\title{
Statin Withdrawal After Major Noncardiac Surgery: Risks, Consequences, and Preventative Strategies
}

\author{
Nabil Fallouh, MD, MS* and Vineet Chopra, MD, MSc
}

Department of General Internal Medicine, University of Michigan Health System, Ann Arbor, Michigan.

BACKGROUND: A growing body of research suggests that statins improve perioperative cardiac outcomes by attenuating inflammation. Conversely, some studies suggest that withdrawal of statins after surgery results in an upsurge of inflammation and adverse cardiac outcomes.

METHODS: We performed a literature search using multiple medical databases to examine the basic, clinical, and experimental evidence supporting the existence of a statin withdrawal state. Studies examining outcomes associated with statin withdrawal were narratively synthesized.

RESULTS: Published evidence suggests that statin withdrawal is associated with worse cardiac outcomes in a variety of scenarios, including acute coronary syndrome, ischemic stroke, and surgery. Although certain reasons for postoperative statin cessation are difficult to avoid (eg, ileus after surgery), we posit that many perioperative clinicians may be unaware of the importance of statin resumption in a timely fashion. This lack of awareness translates into preventable harm and an opportunity for outcome improvement. We introduce innovative practices through which perioperative practitioners may prevent statin discontinuation.

CONCLUSIONS: Ensuring the resumption of statins after surgery should become routine practice for perioperative providers. We highlight knowledge gaps and identify a research agenda aimed at better understanding this practice. Journal of Hospital Medicine 2012;7:573-579. (C) 2012 Society of Hospital Medicine
Accumulating evidence suggests that perioperative treatment with 3-hydroxy-3-methylglutaryl coenzymeA (HMG-CoA) reductase inhibitors (or, "statins") reduces the incidence of cardiovascular events during noncardiac surgery. ${ }^{1-6}$ This evidence has lead the European Society of Cardiology (ESC) and American College of Cardiology Foundation/American Heart Association (ACCF/AHA) to endorse the use of perioperative statins in patients already on this treatment or those at high-risk of cardiovascular events. ${ }^{7,8}$

However, statins are available only in oral formulation. Consequently, prolonged bowel recovery or clinical instability may interfere with use during surgery. Furthermore, many clinicians may not recognize the imperative of postoperative statin resumption, viewing them principally as lipid-lowering entities and not as agents of perioperative benefit. Failure to resume statins postoperatively can be catastrophic, as the ensuing inflammation and thrombosis frequently culminates in myocardial infarction (MI) or death.,

In this article, we review the potent anti-inflammatory properties of statins and their role in preventing perioperative cardiac events. We outline the biochemi-

*Address for correspondence and reprint requests: Nabil Fallouh, MD, MS, Department of General Internal Medicine, University of Michigan Health System, 1500 E Medical Center Dr, SPC 5376, Ann Arbor, Ml 48109; E-mail: nabil@umich.edu

Additional Supporting Information may be found in the online version of this article.

Received: January 18, 2012; Revised: March 23, 2012; Accepted: March 31, 2012

2012 Society of Hospital Medicine DOI 10.1002/jhm.1945

Published online in Wiley Online Library (Wileyonlinelibrary.com). cal basis for perioperative statin benefit, summarizing the basic, clinical, and experimental evidence regarding statin withdrawal. We conclude by presenting strategies to avert postoperative statin cessation and outline a research agenda dedicated to informing this practice.

\section{METHODS}

We performed a literature search using MEDLINE via Ovid (1946-present), EMBASE (1946-present), Biosis (1926-present), and Cochrane CENTRAL (1960-present). We used Boolean logic to search for key terms including statins, 3-hydroxy-3-methylglutaryl CoA reductase inhibitors, death, MI, stroke, acute coronary syndrome (ACS), and statin withdrawal or cessation. All studies published in full-text or abstract form were included. A total of 489 articles were retrieved by this search (last updated March 15, 2012). For this narrative review, we focused on studies that examined adverse outcomes associated with statin withdrawal.

\section{BIOCHEMICAL BASIS OF STATIN PLEIOTROPICITY}

The non-lipid-lowering or "pleiotropic" properties of statins are especially valuable in the perioperative setting. ${ }^{1-6,11}$ Perioperative cardiac complications occur due to oxygen supply:demand mismatch, vascular inflammation, or a combination of these states. A significant perisurgical catecholamine surge produces unopposed sympathetic effects, ${ }^{12}$ increasing the risk of rupture of vulnerable coronary plaques, thrombus formation, and adverse cardiac events. ${ }^{13,14}$ Similarly, augmented inflammatory responses and increased 


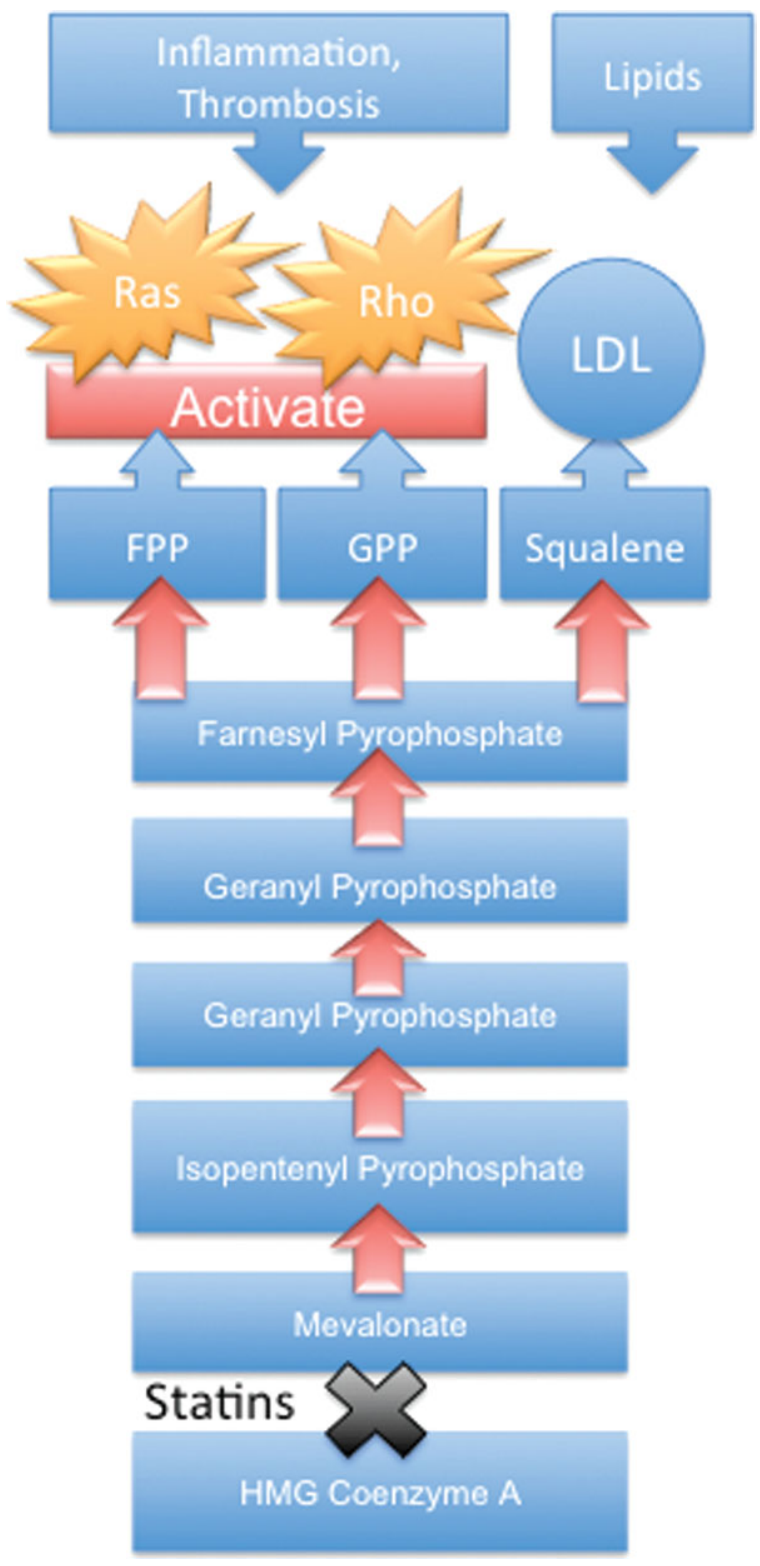

FIG. 1. Pleiotropic effects of statins and statin withdrawal. Statins inhibit 3hydroxy-3-methylglutaryl coenzyme-A (HMG-CoA) reductase to prevent mevalonate formation. Statin withdrawal reverses this phenomenon and upregulates inflammatory molecule production. Abbreviations: FPP, farnesylpyrophosphate; GPP, geranyl-pyrophosphate; LDL, low-density lipoprotein.

circulating coagulation factors further predispose to a hazardous perioperative milieu. ${ }^{15}$ Statins attenuate this vascular inflammatory response by suppressing the synthesis of mevalonate by inhibiting HMG-CoA reductase. Suppression of mevalonate synthesis reduces the bioavailability of 2 important isoprenoid molecules: farnesyl-pyrophosphate and geranylgeranyl-pyrophosphate. ${ }^{16}$ Diminution of these isoprenoid intermediaries leads to reductions in the active intracellular signaling molecules Ras, Rho, and Rac, which play critical roles in vascular reactivity, endothelial function, and coagulation and inflammatory pathways. ${ }^{17-23}$ The cumulative effect of these cellular changes is diminished inflammation during periods of surgical stress (Figure 1).
While the perioperative pleiotropicity of statins is of inherent clinical value, several studies have shown that these effects are lost and even reversed when statins are withdrawn. ${ }^{24-28}$ During statin treatment, absence of isoprenoid intermediaries induces cytosolic accumulation of nonactivated $R h o$ and $R a c$ proteins. Abrupt cessation of statins activates $R h o / R a c$-kinase pathways, leading to unregulated inflammation, platelet hyper-activation, and endothelial dysfunction. ${ }^{24,25,28,29}$ For instance, statin withdrawal in micemodels leads to an "overshoot" activation of Rho, resulting in down-regulation of endothelial nitric oxide production, ${ }^{25}$ activation of nicotinamide adenine dinucleotide phosphate (NAD $[\mathrm{P}] \mathrm{H})$-oxidase, and increased superoxide production. ${ }^{29}$ In another mousemodel, statin withdrawal was associated with up-regulation of key pro-thrombotic molecules including platelet factor 4 and beta-thromboglobulin. ${ }^{24}$ In human studies, a platelet "hyper-activation state" (manifested by increased platelet P-selectin expression and enhanced platelet aggregation) occurs after statin discontinuation. ${ }^{27}$ Furthermore, withdrawal of statins in patients with hyperlipidemia increases inflammatory markers such as C-reactive protein and interleukin$6{ }^{26}$ In the perioperative context, absence of these important anti-inflammatory properties increases the risk of cardiac events. ${ }^{9,10}$

\section{EVIDENCE SUGGESTING BENEFIT FROM PERIOPERATIVE STATIN TREATMENT}

Retrospective studies first suggested clinical benefit from perioperative statin treatment. In a case-control study involving 2816 patients undergoing vascular surgery at Erasmus Medical Center, statin use was associated with substantially decreased postoperative mortality (adjusted odd ratio [OR] 0.22, 95\% confidence interval [CI] $0.10-0.47) .{ }^{5}$ In a subsequent retrospective cohort study of 780,591 patients who underwent major noncardiac surgery, the risk of postoperative mortality was considerably lower among statin users (unadjusted OR $0.68,95 \%$ CI $0.64-0.72$ ) compared to patients who did not receive, or received delayed treatment with statins. ${ }^{3}$ A third retrospective study of 1163 vascular surgery patients found that statins prevented perioperative cardiac complications including death, MI, congestive heart failure, and ventricular tachyarrhythmias (OR 0.52 , 95\% CI 0.35 $0.76){ }^{4}$

The benefit from statin treatment found in retrospective studies prompted the first double-blinded, randomized controlled trial (RCT) of perioperative statin use. In 2004, Durazzo and colleagues ${ }^{1}$ randomized 100 statin-naive patients scheduled to undergo elective aortic, femoro-popliteal, or carotid surgery to receive either $20 \mathrm{mg}$ of atorvastatin or placebo for 45 days. Vascular surgery was performed, on average, 31 days after randomization. Atorvastatin therapy reduced the incidence of death from cardiac causes, 
nonfatal acute MI, ischemic stroke, and unstable angina $26 \%$ in the placebo group vs $8 \%$ in the atorvastatin group; $P=0.031) .{ }^{1}$ Although the small size of the trial rendered it underpowered to show a mortality benefit, this remains the first RCT to demonstrate a protective perioperative effect of statins.

In the 2009 Dutch Echocardiographic Cardiac Risk Evaluation Applying Stress Echocardiography (DECREASE)-III trial, Schouten and colleagues ${ }^{6}$ randomized 497 high-risk, statin-naive patients undergoing vascular surgery to receive, in addition to a beta-blocker, either fluvastatin or placebo before surgery (median of 37 days). Postoperative myocardial ischemia (hazard ratio [HR] $0.55,95 \%$ CI $0.34-0.88$ ), and combined death from cardiovascular causes or nonfatal MI (HR 0.47, 95\% CI 0.24-0.94), occurred less frequently in the treatment group. ${ }^{6}$ In 2009, the same group published DECREASE-IV, a multicenter, prospective, open-label, $2 \times 2$ factorial design trial of 1066 intermediate-risk patients, scheduled to undergo elective, noncardiac surgery. Patients were assigned to bisoprolol, fluvastatin, combination treatment, or control therapy before surgery (median of 34 days). Although those randomized to fluvastatin demonstrated lower incidence of 30-day cardiac death and MI than control (HR 0.65, 95\% CI 0.35-1.10), these outcomes failed to reach statistical significance as the trial was principally powered to examine the effects of perioperative beta-blockade. ${ }^{30}$

Using this pool of data, a meta-analysis of 15 studies $(223,010$ patients) found a substantial $38 \%$ reduction in the risk of mortality after cardiac surgery $(1.9 \%$ vs $3.1 \% ; P=0.0001)$ and an even greater $59 \%$ reduction in the risk of mortality following vascular surgery $(1.7 \%$ vs $6.1 \% ; P=0.0001)$ with perioperative statin therapy. When including noncardiac surgery, a $44 \%$ reduction in mortality was observed $(2.2 \%$ vs $3.2 \% ; P<0.01) .{ }^{2}$ We performed a similar meta-analysis of 15 RCTs involving 2292 patients to determine whether perioperative statin treatment in statin-naive patients, undergoing either cardiac or noncardiac surgery, improved clinical outcomes. Our analysis also found statistically significant reductions in the risk of MI associated with perioperative statin use in both cardiac and noncardiac surgery (risk reduction [RR] $0.53,95 \%$ CI $0.38-0.74$ ) and atrial fibrillation in statin-naive patients undergoing cardiac surgery (RR $0.56,95 \%$ CI $0.45-0.69$ ). ${ }^{31}$ Taken together, a large volume of evidence supports the use of statins in surgical settings.

In view of this evidence, the ACCF/AHA perioperative guidelines for noncardiac surgery endorsed statins as an important risk-reducing intervention in those undergoing noncardiac surgery, and recommended continued use in patients on chronic statin treatment scheduled for noncardiac surgery (Level of Evidence B, Class I; Benefits $>>>$ Risk). Initiating statins in patients undergoing vascular surgery, with or without risk factors, was considered reasonable (Level of Evidence B, Class IIa; Benefits $>>$ Risk). ${ }^{7}$ Current ESC perioperative guidelines in noncardiac surgery offer similar recommendations to those of ACCF/AHA, but differ by categorizing the recommendation to initiate statins in patients at high cardiovascular risk as a Class I recommendation. ${ }^{8}$

\section{CLINICAL CONSEQUENCES OF STATIN WITHDRAWAL}

Although statins provide important cardiac benefits, an important limitation to their perioperative use remains their oral-only formulation. Thus, patients who are unable to resume oral intake may fail to resume treatment. Perioperative statin cessation has been hypothesized to lead to a "statin withdrawal" phenomenon. The evidence that supports the existence of this phenomenon comes from 3 distinct populations: ACS, ischemic stroke, and perioperative patients (Table 1).

\section{Statin Withdrawal in Acute Coronary Syndromes}

Several studies have demonstrated an association between statin withdrawal and heightened risk of cardiovascular events in ACS. ${ }^{32-34}$ In a retrospective analysis of 1616 patients presenting with ACS, withdrawal of statins during or after admission was associated with more frequent death and nonfatal MI compared to those who continued therapy (OR 2.93, 95\% CI 1.64-6.27). ${ }^{33}$ In another retrospective observational study of 68,606 non-ST-segment elevation MI patients, statin cessation during the first 24 hours of hospitalization was independently associated with adverse outcomes including in-hospital death (adjusted HR 1.83; 95\% CI 1.58-2.13), cardiac arrest, and cardiogenic shock. ${ }^{32}$ In a population-based, cohort study in the United Kingdom, statin cessation following an acute MI was independently associated with greater all-cause mortality at 1-year (adjusted HR 1.88, 95\% CI 1.13-3.07). ${ }^{34}$

The significantly increased risk of adverse outcomes associated with the interruption of statins in ACS may be moderated by vascular inflammation related to the inciting coronary event, as statin discontinuation in patients with stable cardiac conditions was not associated with increased risk of cardiovascular events in a large-scale, double-blind, parallel-group study. ${ }^{35}$

\section{Statin Withdrawal in Ischemic Stroke}

Adverse events associated with statin withdrawal have also been reported in patients with cerebrovascular disease. In a prospective observational study of 631 consecutive stroke survivors, those who discontinued statins (owing to mild adverse effects or unclear reasons) experienced increased mortality during the first year after the event (adjusted HR 2.78, 95\% CI 1.963.72). ${ }^{36}$ Using a controversial study design aimed at evaluating the effects of stopping oral intake 
(including chronic medications) during the first days of acute stroke, Blanco and colleagues ${ }^{37}$ randomized 89 stroke victims on chronic statins to either continue medications or experience statin withdrawal following admission. Statin withdrawal was independently associated with increased risk of mortality and dependency at 3 months (OR 4.66, 95\% CI 1.46-14.91). ${ }^{37}$

\section{Perioperative Statin Withdrawal}

In the perioperative setting, statin withdrawal has also been associated with adverse outcomes. Using a quasiexperimental design, Le Manach et al. ${ }^{9}$ evaluated the risk of cardiac complications after infra-renal aortic surgery when immediate, postoperative resumption of statins was adopted at their institution. The investigators compared the risk of developing MI, cardiac death, or abnormal troponin release in 491 patients who did not get early postoperative statin resumption (pre-intervention group) to 178 patients who did. Statin withdrawal for 4 days was demonstrated to be an independent predictor of postoperative troponin leak and MI (OR 2.9, 95\% CI 1.6-5.5). Similarly, Schouten et al. ${ }^{10}$ investigated the risk of adverse events related to interruption of long-term statins by examining cardiac outcomes in 298 statin users undergoing major vascular surgery. Among the 70 patients who experienced statin withdrawal, an increased risk of postoperative troponin release (HR 4.6, 95\% CI 2.2-9.6), and the composite endpoint of MI and cardiovascular death (HR 7.5, 95\% CI 2.8-20.1), was observed compared to those who resumed treatment. Not unexpectedly, the most common reason for statin cessation was inability to take oral medications after surgery. However, even in patients who discontinued statins, the use of extended-release fluvastatin was associated with fewer perioperative cardiac events than other statins. Furthermore, extended-release fluvastatin was also held for 2 days following surgery (owing to inability to take the drug orally), in $25 \%$ of patients in the DECREASE-III study. However, no impact in the rate of adverse outcomes was noted despite this interruption (OR 1.1, 95\% CI 0.48-2.52). ${ }^{6}$ Although the authors surmised that the extended formulation of fluvastatin had provided sustained levels of statin activity despite lack of timely oral intake, it is important to note that this theory may not be generalizable to chronic statin users, as they were not enrolled in this study. Conversely, some patients may have experienced postoperative ileus for longer than 2 days, perhaps resulting in confounding or attenuation of the effect noted in the study.

\section{CLINICAL INSIGHTS INTO FAILURE OF POSTOPERATIVE STATIN RESUMPTION}

We hypothesize that failure to resume perioperative statins may occur for 4 cardinal reasons. First, resumption of an oral agent frequently proves 


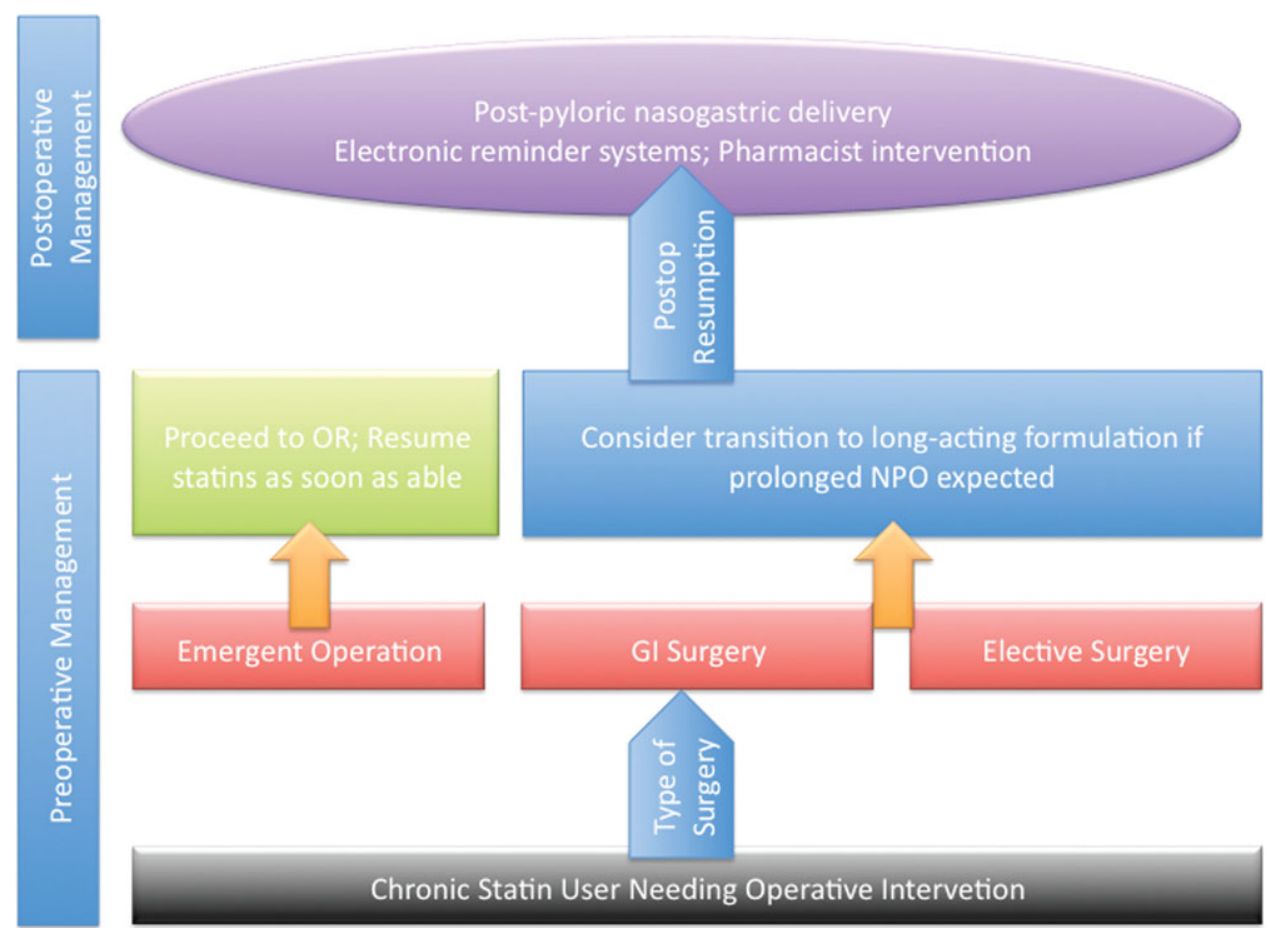

FIG. 2. Clinical strategies to prevent statin withdrawal. Abbreviations: Gl, gastrointestinal; NPO, nil per os (nothing by mouth); OR, operating room.

clinically challenging when complications such as postoperative ileus, nausea, and vomiting peak. To date, no intravenous statin formulations are available, although phase-I studies are currently underway. ${ }^{38}$ Second, it is not inconceivable that perioperative clinical instability may overshadow the resumption of statin treatment. Third, clinicians may also remain concerned regarding adverse effects of statins, a thought compounded by US Food and Drug Administration statin package inserts that specifically advocate for statins to be withheld during surgery. However, although the occurrence of elevated liver function tests and myopathy are theoretically important, the overwhelming majority of perioperative statin studies in noncardiac surgery have not found this to be a major occurrence. ${ }^{39}$ Nonetheless, a lack of uniform definitions and appropriate surveillance for adverse events are important limitations to this finding. In our recent systematic review, we were unable to provide refined estimates of these important side effects owing to differences in definition, variations in screening, and absence of standardized cutoffs used in studies. ${ }^{31}$ Finally, an important reason for failing to resume postoperative statins is that many physicians simply fail to recognize the perioperative importance of these agents.

\section{STRATEGIES TO IMPROVE PERIOPERATIVE STATIN RESUMPTION}

Using the existing evidence, we propose the following 4 clinical strategies to assist in avoiding a statin withdrawal state.

\section{Nasogastric Administration}

Utilizing a post-pyloric nasogastric tube is a straightforward solution to provide statins in those who cannot otherwise tolerate oral intake due to nausea or emesis. Although this solution is hardly innovative, it is relevant as it forces consideration of the need to resume postoperative statins by available means. While the development of a high nasogastric output or a prolonged ileus may limit the applicability of this intervention, it is important that this option be considered as opposed to expectant watching for the clinical return of bowel function. Simvastatin, atorvastatin, rosuvastatin, and pravastatin can be crushed and delivered through this route. ${ }^{40}$

\section{Development of Reminder Systems}

Computerized reminder systems have proved important in ensuring the resumption of deep venous thrombosis prophylaxis and other preventative care compliance in hospitalized patients. ${ }^{41,42}$ Using this process, pharmacist- or electronic health record-based reminder systems could be implemented to ensure that statins are restarted when clinically feasible. Further studies are needed to test whether this approach can lead to improved outcomes.

\section{Medication Reconciliation Prior to Hospital Discharge}

Statin withdrawal highlights the pertinence of a robust, medical reconciliation process prior to the patient's departure from the hospital. In this context, the development of policies using single- or multi-faceted interventions that promote cooperation between 
inpatient physicians, surgeons, and pharmacists with outpatient primary care providers are necessary. ${ }^{43}$

\section{Preoperative Transition to Extended Release Statin Formulations}

An innovative approach to minimizing statin withdrawal involves preoperative transition to an extended-release statin formulation. This strategy may be of particular value in patients where prolonged bowel nonavailability is likely, such as those undergoing gastrointestinal surgery, or when prolonged postoperative dietary restriction (eg, NPO [nil per os]: nothing by mouth) status is expected (Figure 2).

\section{CONCLUSIONS AND FUTURE DIRECTIONS}

Sudden withdrawal of perioperative statins results in adverse clinical outcomes. Individuals engaged in the care of patients during surgery such as hospitalists, anesthesiologists, and surgeons must become more cognizant of a statin withdrawal state.

An important limitation associated with the study of perioperative statin withdrawal remains the ambiguity regarding the extent of the problem in the United States. Therefore, a logical first step could be the use of infrastructure within the National Surgical Quality Improvement Program (NSQIP) to understand the epidemiology of perioperative statin use and consequences associated with statin discontinuation. ${ }^{44}$ Mandating such quality reporting could easily be built into current NSQIP performance metrics. These data would help inform a research agenda targeting patients that experience statin withdrawal and strategies most likely to prevent it.

Disclosure: Nothing to report.

\section{References}

1. Durazzo AE, Machado FS, Ikeoka DT, et al. Reduction in cardiovascular events after vascular surgery with atorvastatin: a randomized trial. I Vasc Surg. 2004;39(5):967-975.

2. Hindler K, Shaw AD, Samuels J, Fulton S, Collard CD, Riedel B. Improved postoperative outcomes associated with preoperative statin therapy. Anesthesiology. 2006;105(6):1260-1272; quiz 1289-1290.

3. Lindenauer PK, Pekow P, Wang K, Gutierrez B, Benjamin EM. Lipidlowering therapy and in-hospital mortality following major noncardiac surgery. JAMA. 2004;291(17):2092-2099.

4. O'Neil-Callahan K, Katsimaglis G, Tepper MR, et al. Statins decrease perioperative cardiac complications in patients undergoing noncardiac vascular surgery: the Statins for Risk Reduction in Surgery (StaRRS) study. J Am Coll Cardiol. 2005;45(3):336-342.

5. Poldermans D, Bax JJ, Kertai MD, et al. Statins are associated with a reduced incidence of perioperative mortality in patients undergoing major noncardiac vascular surgery. Circulation. 2003;107(14): $1848-1851$.

6. Schouten O, Boersma E, Hoeks SE, et al. Fluvastatin and perioperative events in patients undergoing vascular surgery. N Engl J Med. 2009;361(10):980-989.

7. Fleisher LA, Beckman JA, Brown KA, et al. ACC/AHA 2007 guidelines on perioperative cardiovascular evaluation and care for noncardiac surgery: a report of the American College of Cardiology/ American Heart Association Task Force on Practice Guidelines (Writing Committee to Revise the 2002 Guidelines on Perioperative Cardiovascular Evaluation for Noncardiac Surgery): developed in collaboration with the American Society of Echocardiography, American Society of Nuclear Cardiology, Heart Rhythm Society, Society of Cardiovascular Anesthesiologists, Society for Cardiovascular Angiog- raphy and Interventions, Society for Vascular Medicine and Biology, and Society for Vascular Surgery. Circulation. 2007;116(17): e418-e499.

8. Poldermans D, Bax JJ, Boersma E, et al. Guidelines for pre-operative cardiac risk assessment and perioperative cardiac management in non-cardiac surgery. Eur Heart J. 2009;30(22):2769-2812.

9. Le Manach Y, Godet G, Coriat P, et al. The impact of postoperative discontinuation or continuation of chronic statin therapy on cardiac outcome after major vascular surgery. Anesth Analg. 2007;104(6): $1326-1333$.

10. Schouten O, Hoeks SE, Welten GM, et al. Effect of statin withdrawal on frequency of cardiac events after vascular surgery. Am J Cardiol. 2007;100(2):316-320.

11. Davignon J. Beneficial cardiovascular pleiotropic effects of statins. Circulation. 2004:109(23 suppl 1):III39-III43.

12. Roth-Isigkeit A, Brechmann J, Dibbelt L, Sievers HH, Raasch W, Schmucker P. Persistent endocrine stress response in patients undergoing cardiac surgery. J Endocrinol Invest. 1998;21(1):12-19.

13. Dawood MM, Gutpa DK, Southern J, Walia A, Atkinson JB, Eagle KA. Pathology of fatal perioperative myocardial infarction: implications regarding pathophysiology and prevention. Int J Cardiol. 1996; $57(1): 37-44$

14. Devereaux PJ, Goldman L, Cook DJ, Gilbert K, Leslie K, Guyatt GH. Perioperative cardiac events in patients undergoing noncardiac surgery: a review of the magnitude of the problem, the pathophysiology of the events and methods to estimate and communicate risk. Can Med Assoc J. 2005;173(6):627-634.

15. Mangano DT. Perioperative cardiac morbidity. Anesthesiology. 1990;72(1):153-184.

16. Laufs U, Liao JK. Isoprenoid metabolism and the pleiotropic effects of statins. Curr Atheroscler Rep. 2003;5(5):372-378.

17. Laufs U, La Fata V, Plutzky J, Liao JK. Upregulation of endothelial nitric oxide synthase by HMG CoA reductase inhibitors. Circulation. 1998;97(12):1129-1135.

18. Noma K, Goto C, Nishioka K, et al. Roles of rho-associated kinase and oxidative stress in the pathogenesis of aortic stiffness. J Am Coll Cardiol. 2007;49(6):698-705.

19. Sawada N, Itoh H, Ueyama K, et al. Inhibition of rho-associated kinase results in suppression of neointimal formation of balloon-injured arteries. Circulation. 2000;101(17):2030-2033.

20. Seljeflot I, Tonstad S, Hjermann I, Arnesen H. Reduced expression of endothelial cell markers after 1 year treatment with simvastatin and atorvastatin in patients with coronary heart disease. Atherosclerosis. 2002;162(1):179-185.

21. Takemoto M, Node K, Nakagami H, et al. Statins as antioxidant therapy for preventing cardiac myocyte hypertrophy. J Clin Invest. 2001;108(10):1429-1437.

22. Wang CY, Liu PY, Liao JK. Pleiotropic effects of statin therapy: molecular mechanisms and clinical results. Trends Mol Med. 2008; $14(1): 37-44$.

23. Wassmann S, Laufs U, Baumer AT, et al. Inhibition of geranylgeranylation reduces angiotensin II-mediated free radical production in vascular smooth muscle cells: involvement of angiotensin AT1 receptor expression and Rac1 GTPase. Mol Pharmacol. 2001;59(3):646-654.

24. Gertz K, Laufs U, Lindauer U, et al. Withdrawal of statin treatment abrogates stroke protection in mice. Stroke. 2003;34(2):551-557.

25. Laufs U, Endres M, Custodis F, et al. Suppression of endothelial nitric oxide production after withdrawal of statin treatment is mediated by negative feedback regulation of rho GTPase gene transcription. Circulation. 2000;102(25):3104-3110.

26. Li JJ, Li YS, Chu JM, et al. Changes of plasma inflammatory markers after withdrawal of statin therapy in patients with hyperlipidemia. Clin Chim Acta. 2006;366(1-2):269-273.

27. Puccetti L, Pasqui AL, Pastorelli M, et al. Platelet hyperactivity after statin treatment discontinuation. Thromb Haemost. 2003;90(3): 476-482.

28. Sposito AC, Carvalho LS, Cintra RM, et al. Rebound inflammatory response during the acute phase of myocardial infarction after simvastatin withdrawal. Atherosclerosis. 2009;207(1):191-194.

29. Endres M, Laufs U. Effects of statins on endothelium and signaling mechanisms. Stroke. 2004;35(11 suppl 1):2708-2711.

30. Dunkelgrun M, Boersma E, Schouten O, et al. Bisoprolol and fluvastatin for the reduction of perioperative cardiac mortality and myocardial infarction in intermediate-risk patients undergoing noncardiovascular surgery: a randomized controlled trial (DECREASE-IV). Ann Surg. 2009;249(6):921-926.

31. Chopra V, Wesorick DH, Sussman JB, et al. Effect of perioperative statins on death, myocardial infarction, atrial fibrillation, and length of stay: a systematic review and meta-analysis. Arch Surg. 2012; 147(2):181-189.

32. Spencer FA, Fonarow GC, Frederick PD, et al. Early withdrawal of statin therapy in patients with non-ST-segment elevation myocardial infarction: National Registry of Myocardial Infarction. Arch Intern Med. 2004;164(19):2162-2168. 
33. Heeschen C, Hamm CW, Laufs U, Snapinn S, Bohm M, White HD. Withdrawal of statins increases event rates in patients with acute coronary syndromes. Circulation. 2002;105(12):1446-1452.

34. Daskalopoulou SS, Delaney JA, Filion KB, Brophy JM, Mayo NE, Suissa S. Discontinuation of statin therapy following an acute myocardial infarction: a population-based study. Eur Heart J. 2008; 29(17):2083-2091.

35. McGowan MP. There is no evidence for an increase in acute coronary syndromes after short-term abrupt discontinuation of statins in stable cardiac patients. Circulation. 2004;110(16):2333-2335.

36. Colivicchi F, Bassi A, Santini M, Caltagirone C. Discontinuation of statin therapy and clinical outcome after ischemic stroke. Stroke. 2007;38(10):2652-2657.

37. Blanco M, Nombela F, Castellanos M, et al. Statin treatment withdrawal in ischemic stroke: a controlled randomized study. Neurology. 2007;69(9):904-910.

38. Prinz V, Laufs U, Gertz K, et al. Intravenous rosuvastatin for acute stroke treatment: an animal study. Stroke. 2008;39(2):433-438.
39. Schouten O, Kertai MD, Bax JJ, et al. Safety of perioperative statin use in high-risk patients undergoing major vascular surgery. Am J Cardiol. 2005;95(5):658-660.

40. Lexi-Comp Online ${ }^{\mathrm{TM}}$, Lexi-Drugs ${ }^{\mathrm{TM}}$, Hudson, OH: Lexi-Comp, Inc; December 7, 2011.

41. Dexter PR, Perkins S, Overhage JM, Maharry K, Kohler RB, McDonald CJ. A computerized reminder system to increase the use of preventive care for hospitalized patients. N Engl J Med. 2001;345(13): 965-970.

42. Kucher N, Koo S, Quiroz R, et al. Electronic alerts to prevent venous thromboembolism among hospitalized patients. N Engl J Med. 2005; 352(10):969-977.

43. Geurts MM, Talsma J, Brouwers JR, de Gier JJ. Medication review and reconciliation with cooperation between pharmacist and general practitioner and the benefit for the patient: a systematic review. $\mathrm{Br} \mathrm{J}$ Clin Pharmacol. January 13, 2012. doi: 10.1111/j.1365-2125. 2012.04178.x.

44. American College of Surgeons National Surgical Quality Improvement Program. Available at: http://www.acsnsqip.org. Accessed December 15,2012 